value of computed tomography in the search for an unknown primary tumour. ROFO 1988;149:277-9,

$13 \mathrm{Ma} \mathrm{WX}, \mathrm{Li}$ W. Immunohistochemical detection of prostate carcinoma. Chung-hua Ping Li Hsuch 7sa Chih 1989;18:37-9.

14 Sturm U, Grossmann H, Schuh D, Zotler S, Muller M. Immunohistochemical detection of thuroglobulin. Zentralbl Allg Pathol 1989;135:281-5.

15 Hitchcock A, Ellis IO. A combined serological and immunohistological investigation of $\mathrm{CA} 125$ antigen and malignant ovarian epithelial tumours. fObstet Gymaecol 1987:8:173-8.

16 Berchuck A, Soisson AP, Clarke-Pearson DL, Soper JT, Boyer CM, Kinney $\mathrm{BB}$. Immunohistochemical expression of $\mathrm{CA} 125$ in endometrial adeno- carcinoma: correlation of antigen expression with metastatic potential. Cancer Res 1989;49:2091-5.

17 Gendler S, Taylor-Papadimitriou J, Dubig T. A highly immunogenic region of a human polymorphic epithelial mucin expressed by carcinomas made up of tandem repeats. F Biol Chem 1988;263:12820-3.

18 Ellis IO, Robins RA, Elston CW, Blamey RW, Ferry B, Baldwin RW. A monoclonal antibody, NCRC 11, raised to human breast carcinom. 1. Production and immunohistological characterisation. Histopatholo 1984;8:501-16

(Accepted 26 November 1992)

\title{
Who's afraid of informed consent?
}

\section{D Kerrigan, R S Thevasagayam, T O Woods, I Mc Welch, W E G Thomas, A J Shorthouse, A R Dennison}

\begin{abstract}
Objective-To test the assumption that patients will become unduly anxious if they are given detailed information about the risks of surgery in an attempt to obtain fully informed consent.

Design-Preoperative anxiety assessed before and after patients were randomly allocated an information sheet containing either simple or detailed descriptions of possible postoperative complications.
\end{abstract}

Setting-Four surgical wards at two Sheffield hospitals.

Subjects-96 men undergoing elective inguinal hernia repair under general anaesthesia.

Main outcome measure-Change in anxiety level observed after receiving information about potential complications.

Results-Detailed information did not increase patient anxiety (mean Spielberger score at baseline $33.7(95 \%$ confidence interval 31.3 to $36 \cdot 2)$, after information $34.8(32.1$ to $37 \cdot 5) ; p=0 \cdot 20$, paired $t$ test). A simple explanation of the facts provided a statistically significant degree of reassurance (mean score at baseline $34.6(31.5$ to 37.6$)$, after information $32.3(29.8$ to 34.9$) ; p=0.012)$, although this small effect is likely to be clinically important only in those whose baseline anxiety was high $(r=0.27, p=0.05)$.

Conclusions-In men undergoing elective inguinal hernia repair a very detailed account of what might go wrong does not increase patient anxiety significantly and has the advantage of allowing patients a fully informed choice before they consent to surgery, thus reducing the potential for subsequent litigation.

Royal Hallamshire Hospital, Sheffield S10 2JF D D Kerrigan, lecturer R S Thevasagayam, medical student

I Mc Welch, house officer W E G Thomas, consultant surgeon

A J Shorthouse, consultant surgeon

A R Dennison, senior registrar

Department of Child and Adolescent Psychiatry, Booth Hall Children's Hospital, Manchester M9 2AA

T O Woods, senior registrar

Correspondence to: Mr D D Kerrigan, Department of Surgery, Royal Oldham Hospital, Oldham OL1 2JH

BMF 1993;306:298-300

\section{Introduction}

The NHS Management Executive's recent guidance on obtaining consent from patients is a pertinent reminder of the importance with which the government views our legal requirement to obtain fully informed consent from patients undergoing treatment. ${ }^{1}$ In the unhappy event of litigation, a signed consent form may be disregarded by the courts unless it can be shown that the patient was "given sufficient information, in a way they can understand, about the proposed treatment." Sadly, the standards of consent actually achieved on the ward often fall short of those expected by lawyers, perhaps because the task of obtaining consent is left to more junior medical staff, who are themselves ignorant of many of the potential pitfalls that might face the patient. It has been estimated that every year about 300000 patients in the United Kingdom experience some form of harm as a result of being admitted to hospital, ${ }^{2}$ and if claims for medical negligence are to be minimised it is vitally important that doctors ensure that the patient has carefully considered the potential risks of any procedure as well as its likely benefits.

One solution is to adopt the North American practice of providing patients with a comprehensive list of postoperative complications, but to most British doctors the prospect of burdening patients with "unwanted" information about what might go wrong is deemed to be both unhelpful and unkind. In view of this dichotomy of opinion it is surprising that there is little, if any, objective evidence to support or refute the rather paternalistic British view that allowing patients to make a fully informed decision about their treatmen would generate an unnecessary and harmful degree of anxiety. The aim of this study was to find out who is really afraid of fully informed consent: British patients or their doctors?

\section{Subjects and methods}

Ninety six male patients admitted to four surgical wards for elective repair of inguinal hernias under general anaesthesia were interviewed in hospital on the day before surgery and asked to complete two self evaluation questionnaires: a screen for pre-existing anxiety or depressive states using the hospital anxiety and depression scale (HADS), ${ }^{3}$ in which patients were asked to score answers based on how they generally fel over the few weeks before admission, and an assessment of their current state of anxiety (Spielberger STAI-X1). The Spielberger anxiety scale consists of twenty statements that gauge how respondents feel "right now, at this moment" and has been widely evaluated in healthy American adults and in nonpsychiatric hospital inpatients. ${ }^{+}$Subjects chose from one of four graded responses to each statement, generating a total score between 20 (low anxiety) and 80 (very high anxiety). After this baseline assessment was obtained patients were randomly allocated one of two typed information sheets which contained a simple description of what a hernia is, why surgery was necessary, and what the operation entailed. The difference between the two information sheets was that one provided a rather sketchy outline of possible postoperative complications (derived from a survey of what 10 house officers actually told hernia patients when they obtained consent), and the other contained a more comprehensive list (boxes). To prevent patients on the same ward comparing the contents of different fact sheets (and thereby contaminating the data), randomisation depended on the ward and week of admission: forms used on a given ward were randomly alternated each week.

To standardise the manner in which information was presented by the investigator, patients were simply left to digest the written information for one hour, but they were given the opportunity to ask the investigator to 


\section{Simple information sheet}

\section{Complications of inguinal hernia repair}

1 Pain and discomfort in the first few weeks after surgery. You will be supplied with painkillers to help with this

\section{Recurrence of your hernia}

3 Problems with the anaesthetic. Fortunately these are rare. You will be seen by an anaesthetist before your operation. $\mathrm{He} / \mathrm{sh}$ will decide on your fitness to receive the anaesthetic.

clarify anything which they did not understand before their state of anxiety was reassessed by a second Spielberger questionnaire. In addition to the in hospital assessment, we also wanted to know what the patients thought about the amount of information they were given, and to do this we administered a telephone questionnaire consisting of six questions about their inpatient experiences, one of which read "Was the amount of information you received before your operation too much, about right, or too little?"

\section{STATISTICAL ANALYSIS}

The significance of changes in anxiety noted was analysed by Student's paired $t$ tests, and data from the telephone questionnaire were compared by a two tailed Fisher's exact test. The relation between an individual's baseline anxiety and the change in anxiety observed after receiving information was examined by using scatter plots and by calculating the Pearson correlation coefficient as suggested by Bland and Altman $^{5}$ (change in anxiety $v$ average of first and second Spielberger scores).

We estimated that a change of five points in the Spielberger score might be clinically significant; to detect this with $95 \%$ power (assuming a standard deviation of 6 ) the required sample size was calculated to be 40-50 patients in each group.

Patients were simply told that we were aware that emotions could influence how they reacted to illness and were asked to participate in a survey investigating their response to hospital admission for minor surgery. They were not aware that they were taking part in a randomised comparison of two different information sheets as this would have invalidated the study, which received ethical approval from the Royal Hallamshire Hospital ethical committee.

\section{Results}

Table I describes the general characteristics of the two groups studied. Ninety six patients were randomised, with roughly equal numbers receiving each of the

TABLE I-Patient characteristics $(n=96)$. Values are mean $(95 \%$ confidence interval)

\begin{tabular}{lcc}
\hline & \multicolumn{2}{c}{ Information sheet given } \\
\cline { 2 - 3 } & $\begin{array}{c}\text { Simple } \\
(\mathrm{n}=51)\end{array}$ & $\begin{array}{c}\text { Detailed } \\
(\mathrm{n}=45)\end{array}$ \\
\hline $\begin{array}{l}\text { Age (years) } \\
\text { Hospital anxiety and depression score: } \\
\text { Depression } \\
\text { No with score } \geqslant 10\end{array}$ & $59(54$ to 63$)$ & $53(48$ to 58$)$ \\
$\begin{array}{l}\text { Anxiety } \\
\text { No with score } \geqslant 10\end{array}$ & $2 \cdot 2(1.5$ to $2 \cdot 8)$ & $2 \cdot 1(1.5$ to $2 \cdot 7)$ \\
& 0 & 0 \\
& $5.5(4.7$ to $6 \cdot 3)$ & $5.4(4.5$ to 6.3$)$ \\
& 3 & 3
\end{tabular}

TABLE II-Change in anxiety score after receiving information about possible complications. Values are mean ( $95 \%$ confidence interval)

\begin{tabular}{lccc}
\hline Information sheet & Baseline & After information & Mean change \\
\hline Simple & $34.6(32$ to 38$)$ & $32.3(30$ to 35$)$ & $-2 \cdot 2(-3.9$ to -0.5$)$ \\
Detailed & $33.7(31$ to 36$)$ & $34.8(32$ to 38$)$ & $1.07(-0.6$ to $2 \cdot 7)$ \\
\hline
\end{tabular}

two information sheets. The patients were well matched for age and preadmission state of anxiety and depression; the proportion of patients whose score on the hospital anxiety and depression scale was 10 or greater (a level that indicates clinically significant anxiety or depression states) was equal in each study group. The change in anxiety invoked by reading about the potential complications of surgery is shown in table II. There was no change in anxiety in the group who had received detailed information $(p=0 \cdot 20)$, whereas patients who received a rather superficial explanation of the risks were significantly less anxious afterwards $(p=0.012)$, particularly if they had scored highly on the baseline anxiety assessment (fig 1; $r=$ $0 \cdot 27, p=0 \cdot 05)$. There was no evidence to suggest that anxious patients who had been given detailed informa-

\section{Detailed information sheet}

\section{Complications of inguinal hernia repair}

1 Pain and discomfort in the first few weeks after surgery. You will be supplied with painkillers to help with this. It is common for some bruising to occur around the wound, base of the penis and testicles. A serious wound infection occurs in 1 in every 100 patients ( 3 in 100 if the operation is for a recurrent hernia) and other wound problems such as a deep blood clot (haematoma) or separation of the skin and underlying tissues can also occur. Although wound discomfort is usually fairly short lived, nerve injury during the operation occurs after somewhere between 1 in 100 and 1 in 10 hernia operations and this can lead to more persistent or even permanent pain, discomfort or numbness over the groin, the base of the penis, and the upper part of the thigh.

2 A recurrence of your hernia. The chances of this happening for a single hernia (one side) vary from $1 \%$ to $7 \%$, that is $1-7$ patients in every hundred. For a double hernia (both sides) the risk is $2-4 \%$. For a recurrent (re-do) hernia the chances are even higher (somewhere between a 1 in 20 and a 1 in 3 chance of future trouble).

3 Problems with the anaesthetic. Fortunately these are rare. You will be seen by an anaesthetist before your operation. $\mathrm{He} /$ she will decide on your fitness to receive the anaesthetic. Chest problems such as pneumonia and disorders of the heart rhythm and blood pressure are the most common serious problems associated with receiving an anaesthetic.

4 Temporary difficulty with passing urine after surgery occurs in about one third of hernia patients. In some cases, this needs to be relieved by placing a drain (catheter) in the bladder for a few days. Insertion of a catheter can cause injury to the passageway between the bladder and the penis.

5 Permanent damage to a testicle (either loss or permanent shrinkage) can occur in about 3 of every 200 patients undergoing a hernia repair. In addition, the tube(s) carrying sperm from the testicle(s) to the penis might accidentally be cut, which could cause sterility.

6 Occasionally, injury to the intestine or other abdominal organs occurs. This may require repair or removal of the damaged part.

7 Injury to the blood supply of the leg (major artery and vein), which if severe may result in heavy bleeding, blood clot formation or permanent leg swelling with possible skin ulceration.

IN ADDITION THERE ARE CERTAIN OTHER COMPLICATIONS WHICH MAY OCCUR AFTER ANY OPERATION. These include DEEP VEIN THROMBOSIS (a blood clot in the leg which may cause swelling, pain, and PULMONARY EMBOLUS, a blood clot on the lung producing breathing difficulty and possibly death). ALLERGIC REACTIONS can occur to surgical dressings, cleansing agents, and antibiotics. 
tion became more distressed afterwards (fig $2 ; \mathrm{r}=0 \cdot 18$, $\mathrm{p}=0 \cdot 23$ ).

We were able to contact $72 \%(69 / 96)$ of patients after discharge from hospital. Nearly one quarter (8/33) of those who had received the more comprehensive list of complications thought that they had been given "too much" information, compared with just $6 \%(2 / 36)$ of those who had been given a simple explanation $(p=$ $0 \cdot 04$, Fisher's exact test): anxiety scores did not differ significantly between patients who thought the amount of information given was too much and those who thought it was "about right" (data not shown). Only two patients thought that they had not been told enough, even though one of them had been given the very detailed sheet. All 96 patients consented to surgery.

\section{Discussion}

The rate at which compensation claims are being lodged against medical practitioners may be escalating faster in the United Kingdom than in North America, and there are fears that if this trend continues unchecked the cost of litigation may ultimately damage the quality of healthcare by eroding already restrained budgets. $^{7}$ The defence societies constantly warn that most complaints are provoked by doctors failing to communicate adequately, ${ }^{8}$ so there is an argument for adopting the "defensive" practice of our American colleagues when consent for treatment is sought: but

FIG 1-Relation between baseline anxiety and change in anxiety score in 51 patients who received a simple explanation of operative risks $(r=0 \cdot 27$, $p=0.05$ )
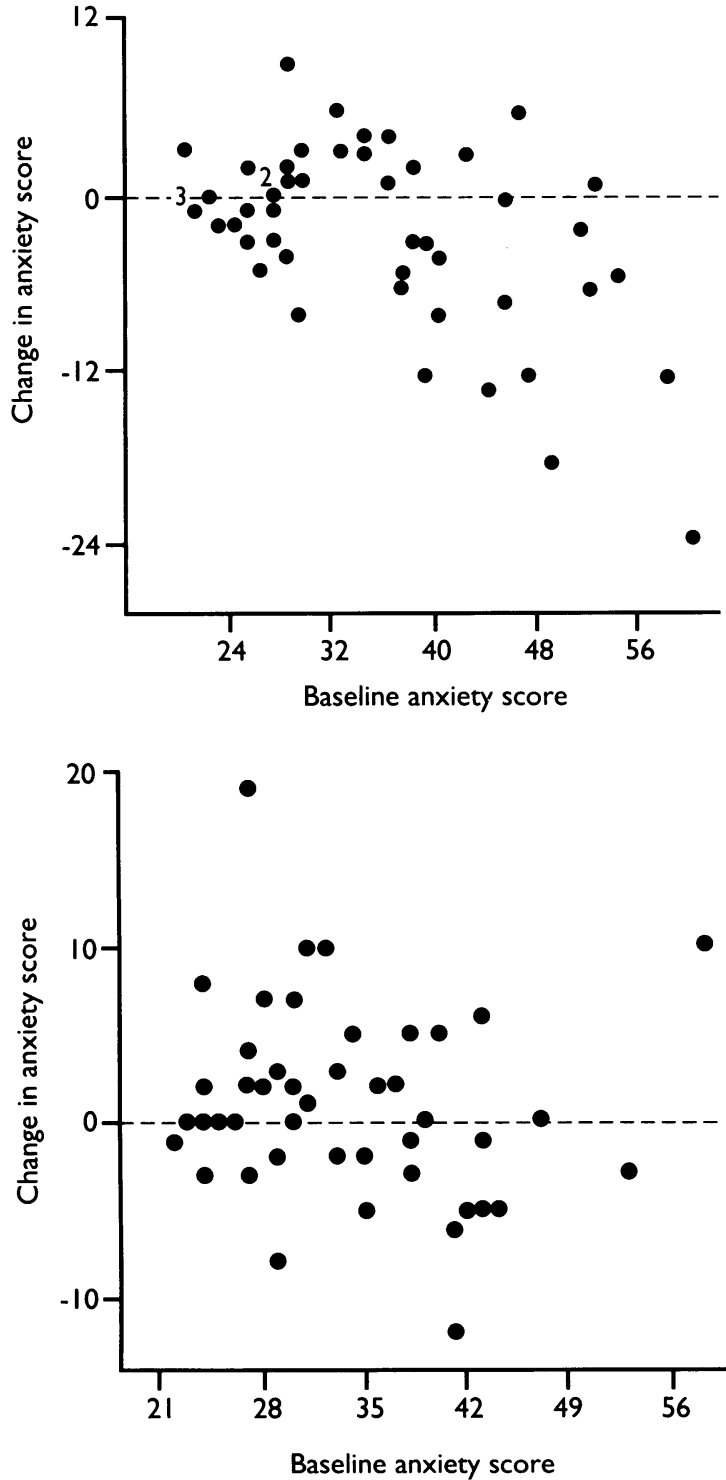

do our patients have the stomach for an American approach?

In an attempt to answer this simple question we used a self administered questionnaire (Spielberger STAI$\mathrm{X} 1$ ) designed to provide an objective index of anxiety at any given moment. All of the findings we report are based on the assumption that this questionnaire can accurately and reproducibly detect a change in anxiety, even when the same questionnaire is administered twice with just one hour between each assessment. In response to this concern Spielberger retested 197 students one hour after they had watched a stressful film depicting gory accidents and concluded that the questionnaire was indeed sensitive to acute changes in anxiety. ${ }^{+}$Our second assumption was that the information given to the patients was digested and understood. There is evidence that at times of stress many patients do not absorb verbal information ${ }^{9}$ and that a better quality of informed consent can be obtained by combining oral and written information as we did. ${ }^{1011}$ To heighten comprehension, our information was presented using personal pronouns in deliberately short sentences that explained or avoided technical terms ${ }^{10}{ }^{12}$; awareness could be further enhanced by issuing the information sheets (customised for specific operations) - during the initial outpatient consultation, which would give patients more time to assimilate the facts.

Our results suggest that it is wrong to assume that patients will become unduly anxious if they are warned about most of the potential risks of surgical treatment, at least as far as inguinal herniorrhaphy and general anaesthesia are concerned. Whether or not the same applies to patients with cancer or those admitted for more complex surgery remains to be seen, but even major operations share many of the "frightening" general risks associated with inguinal hernia repair. Patients are entitled to receive accurate information about their treatment and the risks we ask them to take; they also have a right to withhold consent from such treatment if they feel unhappy about accepting these risks. A full explanation of the facts allows them to make a fully informed decision about their surgery; this might reduce the number of cases of litigation arising from misunderstandings about the purpose and nature of any planned treatment. Against this one has to balance the fact that a simple explanation of the common problems encountered seems to (statistically) reassure the patient, particularly the very anxious individual, although the overall effect was very small and probably clinically insignificant.

What is certain is that as the relationship between doctors and their patients becomes less paternalistic, so must the manner in which we seek to obtain their consent for treatment.

We thank Brian Farragher, department of medical statistics, University Hospital of South Manchester, for advice about presentation of data.

1 Delamothe A. Consenting patients. BMF 1990;301:510.

2 Smith R. The epidemiology of malpractice. $B M \mathcal{F} 1990 ; 301: 621-2$.

3 Zigmond A, Snaith R. The hospital anxiety and depression scale. Acta Psychiatry Scand 1983;67:361-70.

4 Spielberger CD, Gorsuch RL, Lushene R, Vagg PR, Jacobs GA. Manual for the state-trait anxiety inventory (form $Y$ ). Palo Alto, California: Consulting Psychologists Press, 1983

5 Bland JM, Altman DG. Statistical methods for assessing agreement between two methods of clinical measurement. Lancet 1986;i:307-10.

Tribe DMR, Korgaonkar GJA. Liability and litigation trends. $\mathrm{Br}$ f Hosp Med 1990;43:217-20.

7 Black N. Medical litigation and the quality of care. Lancet 1990;335:35-7.

8 Orr CJB. How to minimise litigation. Br F Hosp Med 1989;42:439.

8 Orr CJB. How to minimise litigation. Br F Hosp Med 1989.

10 Edwards MH. Satisfying patients' needs for surgical information. Br $\mathcal{F}$ Surg 1990;77:463-5.

11 Lynöe N, Sandlund M, Dahlqvist G, Jacobsson L. Informed consent: study of quality of information given to participants in a clinical trial. $B M J$ 1991;303:610-3.

12 Bunker TD. Information leaflets for surgical patients. Ann R Coll Surg Engl 1983;65:242-3.

(Accepted 20 November 1992) 